2012

\title{
Metastable Atom And Electron Density Diagnostic In The Initial Stage Of A Pulsed Discharge In Ar And Other Rare Gases By Emission Spectroscopy
}

S. F. Adams

E. A. Bogdanov

V.I. Demidov

M.E. Koepke

A. A. Kudryavtsev

See next page for additional authors

Follow this and additional works at: https://researchrepository.wvu.edu/faculty_publications

\section{Digital Commons Citation}

Adams, S. F.; Bogdanov, E. A.; Demidov, V. I.; Koepke, M. E.; Kudryavtsev, A. A.; and Williamson, J. M., "Metastable Atom And Electron Density Diagnostic In The Initial Stage Of A Pulsed Discharge In Ar And Other Rare Gases By Emission Spectroscopy" (2012). Faculty Scholarship. 595.

https://researchrepository.wvu.edu/faculty_publications/595

This Article is brought to you for free and open access by The Research Repository @ WVU. It has been accepted for inclusion in Faculty Scholarship by an authorized administrator of The Research Repository @ WVU. For more information, please contact ian.harmon@mail.wvu.edu. 
Authors

S. F. Adams, E. A. Bogdanov, V. I. Demidov, M. E. Koepke, A. A. Kudryavtsev, and J. M. Williamson 


\section{AIP Plasmas}

\section{Metastable atom and electron density diagnostic in the initial stage of a pulsed discharge in Ar and other rare gases by emission spectroscopy}

S. F. Adams, E. A. Bogdanov, V. I. Demidov, M. E. Koepke, A. A. Kudryavtsev et al.

Citation: Phys. Plasmas 19, 023510 (2012); doi: 10.1063/1.3686142

View online: http://dx.doi.org/10.1063/1.3686142

View Table of Contents: http://pop.aip.org/resource/1/PHPAEN/v19/i2

Published by the American Institute of Physics.

\section{Related Articles}

Argon plasma modeling with detailed fine-structure cross sections

J. Appl. Phys. 111, 053307 (2012)

Optical and kinetic properties of the dusty plasma in radiofrequency discharge

Phys. Plasmas 19, 023706 (2012)

Continuous wave cavity ring down spectroscopy measurements of velocity distribution functions of argon ions in a helicon plasma

Rev. Sci. Instrum. 83, 023508 (2012)

Laser measurement of $\mathrm{H}$ - ions in a field-effect-transistor based radio frequency ion source

Rev. Sci. Instrum. 83, 02 A731 (2012)

First measurement of electron temperature from signal ratios in a double-pass Thomson scattering system Rev. Sci. Instrum. 83, 023507 (2012)

\section{Additional information on Phys. Plasmas}

Journal Homepage: http://pop.aip.org/

Journal Information: http://pop.aip.org/about/about_the_journal

Top downloads: http://pop.aip.org/features/most_downloaded

Information for Authors: http://pop.aip.org/authors

\section{ADVERTISEMENT}

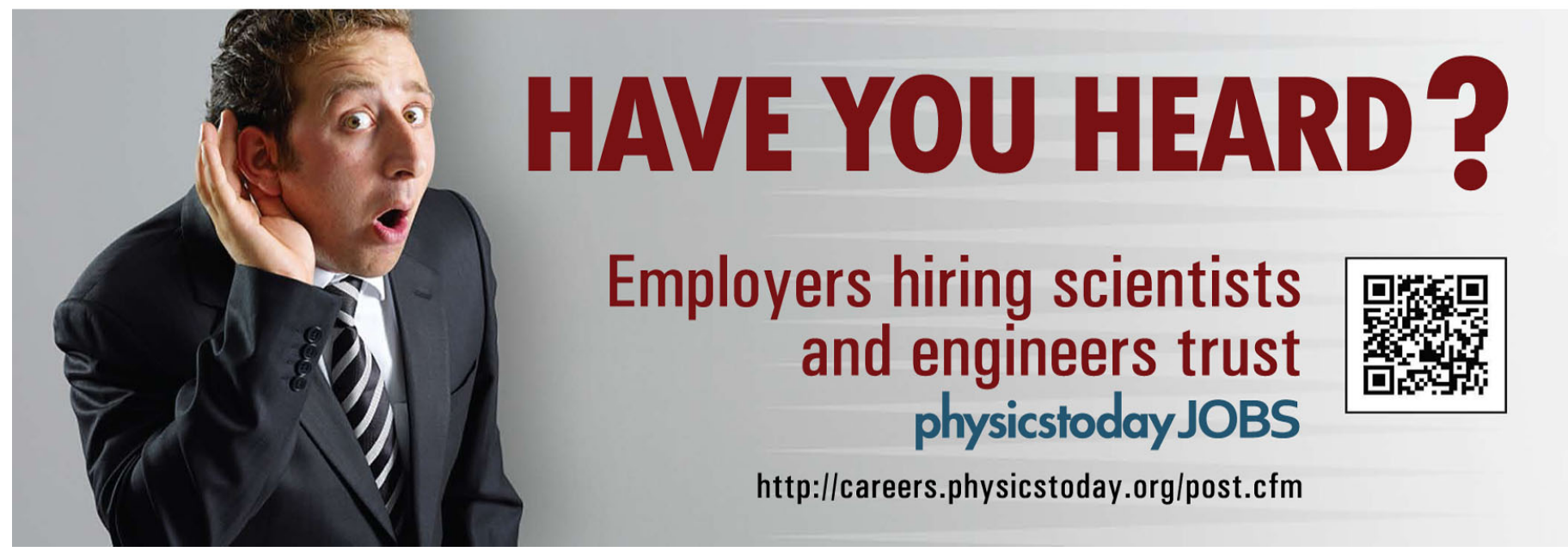




\title{
Metastable atom and electron density diagnostic in the initial stage of a pulsed discharge in Ar and other rare gases by emission spectroscopy
}

\author{
S. F. Adams, ${ }^{1}$ E. A. Bogdanov, ${ }^{2}$ V. I. Demidov, ${ }^{3}$ M. E. Koepke, ${ }^{3}$ A. A. Kudryavtsev, ${ }^{2}$ \\ and J. M. Williamson ${ }^{4}$ \\ ${ }^{1}$ Air Force Research Laboratory, Wright-Patterson AFB, Ohio 45433, USA \\ ${ }^{2}$ St. Petersburg State University, St. Petersburg, Russia \\ ${ }^{3}$ West Virginia University, Morgantown, West Virginia 26506, USA \\ ${ }^{4} U E S$, Inc., 4401 Dayton-Xenia Rd., Dayton, Ohio 45432, USA
}

(Received 22 December 2011; accepted 23 January 2012; published online 23 February 2012)

Temporal measurements of the emission intensities of the Ar 419.8 and $420.1 \mathrm{~nm}$ spectral lines combined with Ar plasma modeling were used to examine the metastable atom and electron density behavior in the initial stage of a pulsed dc discharge. The emission intensity measurements of these spectral lines near the start of a pulsed dc discharge in Ar demonstrated a sharp growth of metastable atom and electron densities which was dependent on the applied reduced electric fields. For lower electric fields, the sharp growth of metastable atom density started earlier than the sharp electron density growth. The reverse situation was observed for larger electric fields. This presents the possibility for controlling plasma properties which may be useful for technological applications. Similar measurements with spectral lines of corresponding transitions in other rare gases are examined. (C) 2012 American Institute of Physics. [doi:10.1063/1.3686142]

\section{INTRODUCTION}

The development of simple and reliable plasma diagnostics, that can provide more detailed information about plasma conditions, is essential for understanding plasma properties and further development of plasma engineering. ${ }^{1,2}$ Thus, many studies describing the development and implementation of different types and modifications of plasma diagnostics can be found in the literature. Principles among these studies are various types of optical spectroscopy (some recent examples are Refs. 3-7) and Langmuir probes (see, for example, Refs. 8-10). This paper is devoted to the implementation of a simple emissive spectroscopy technique for diagnostics of metastable atom, $N_{m}$, and electron, $N_{e}$, densities in the initial stage of a pulsed dc glow discharge in a rare gas. At the beginning stage of a pulsed discharge, the metastable, $N_{m}$, and electron, $N_{e}$, density build-up may be abrupt. ${ }^{11,12}$ The variation of electric field applied to the discharge can impact the ratio between different rate constants which govern plasma physical-chemical reactions which in principle could change the dynamics between the temporal behavior of metastable atom and electron densities. Different temporal behavior of metastable and electron densities in the discharge could create a situation that would be useful for regulating of plasma properties, including the electron energy distribution function (EEDF), for example, due to the creation of energetic electrons in plasma reactions such as Penning ionization. ${ }^{13}$ The regulation of the charged particle distribution functions in plasmas may be important in many technological applications and therefore attracts the attention of plasma researchers (see, e.g., Refs. 14 and 15). While this paper does not intend to examine plasma phenomena and their corresponding effects, for example, the creation of fast electrons arising from Penning ionization, it will give a simple experimental demonstration of the change in relative electron and metastable densities dynamics. Experimental demonstration of the effect in other rare gases is also presented.

In this paper, optical emission spectroscopy is used to measure the metastable atom and electron density build-up in the initial stage of a pulsed dc discharge. The measurements are obtained from the temporal behavior of 419.8 and $420.1 \mathrm{~nm}$ Ar line intensity emission in an Ar discharge. The possibility of utilizing these lines for relative metastable atom/electron density diagnostics in plasmas was previously suggested by DeJoseph and Demidov, ${ }^{16}$ where the emission intensity from 419.8 to $420.1 \mathrm{~nm}$ Ar lines for the qualitative analysis of a pulsed, $\mathrm{rf}$ power discharge was described. Later, Jung et al ${ }^{17}$ measured the corresponding Ar cross-sections enabling more precise quantitative diagnostics. Adams et al. ${ }^{18,19}$ have demonstrated that relative intensity measurements of the spectral lines can provide information about absolute Ar metastable atom density in plasmas.

In Sec. II, modeling results of the direct and stepwise excitation rates for Ar lines 419.8 and $420.1 \mathrm{~nm}$ as well as the dependence of their intensity ratio on the reduced electric field $E / N$ and relative electron and metastable atom densities, $N_{e} / N$, and $N_{m} / N$, respectively are presented. Here, $N$ is the density of ground state atoms. The modeling results can be used for analysis of the Ar spectral lines 419.8 and $420.1 \mathrm{~nm}$ in specific plasma situations. In Sec. III, a simple global model for the initial stage of a pulsed dc discharge is described. The model demonstrates the fast growth of $N_{e}$ and $N_{m}$ and describes the potential control of the relative electron and metastable atom growth by the dependence on $E / N$. Section IV describes the experimental set-up with results of experiments in Ar, Ne, and Xe presented in Sec. V. Conclusions are given in Sec. VI. 


\section{EMISSION LINE RATIO METHOD FOR ARGON PLASMA DIAGNOSTICS}

The 419.8 and $420.1 \mathrm{~nm}$ Ar lines originate from $3 p_{5}$ and $3 \mathrm{p}_{9}$ (Paschen notation) levels, respectively. A partial energy level diagram, showing the spectral lines, is shown in Fig. 1.

The proximity of 419.8 and $420.1 \mathrm{~nm}$ Ar lines makes them very convenient for spectral measurements because this renders additional calibration due to detector and equipment sensitivity unnecessary. At the same time, it may also be important that excitation thresholds for both lines from lower levels are approximately the same.

To derive practical information from the emission line intensities, the population rate constants of the energy levels from various plasma processes are required. Excitation rate constants by electron impact from the lower states were obtained from a numerical model of the Ar plasma. Since the model has been described in detail elsewhere, ${ }^{20}$ only a brief outline will be given here. The model includes direct, stepwise and Penning ionization, excitation of metastable levels, mixing by electron collisions from the metastable to the resonance level and its subsequent radiative deactivation. With the aid of a separate Boltzmann solver, the electron reaction rate constants and electronic transport coefficients were found. The system of charged particles and metastable $\mathrm{Ar}$ atom balance equations and the plasma electron energy transfer equations, taking into account their threedimensional transfer due to the thermal conductivity was solved self-consistently with the electric field and potential obtained from Poisson's equation.

Figure 2 shows electron optical excitation rate constants to the upper level for $420.1 \mathrm{~nm}\left(K_{1 a}\right)$ and $419.8 \mathrm{~nm}\left(K_{2 a}\right) \mathrm{Ar}$ emission lines from the ground state, obtained from the numerical plasma model using the cross-sections of Jung et al. ${ }^{17}$

Excitation rates from $\mathrm{Ar}$ metastable state, $3 \mathrm{p}^{5}, 4 \mathrm{~s}^{3}, \mathrm{P}^{2}$, $K_{1 m}$ for $420.1 \mathrm{~nm}$ and $K_{2 m}$ for $419.8 \mathrm{~nm}$ are also shown. From the figure, it is seen that both lines have almost equal

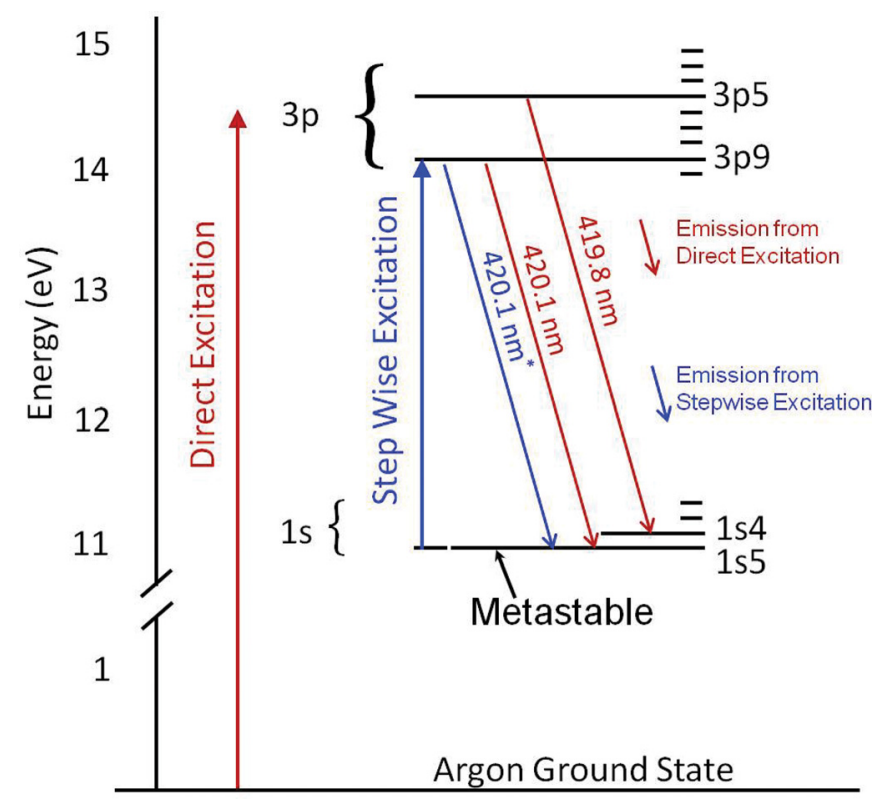

FIG. 1. (Color online) Ar energy level diagram with excitation to the $3 p$ states.

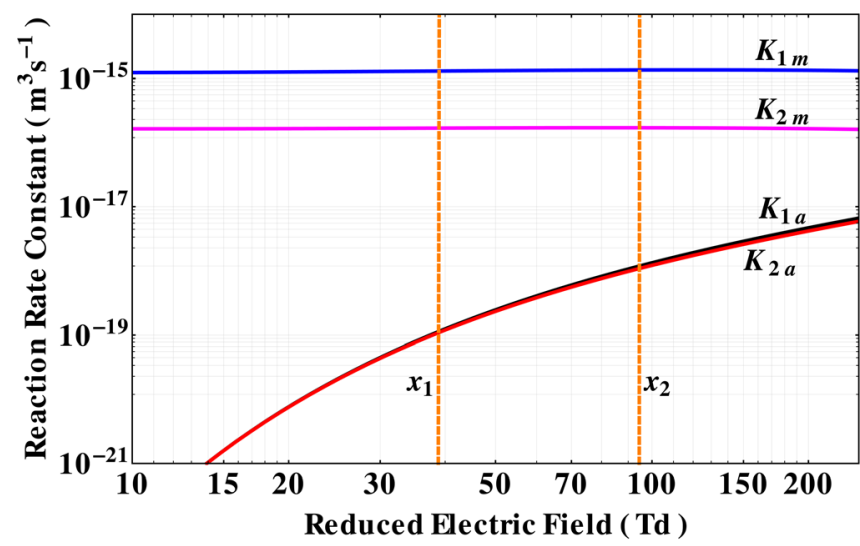

FIG. 2. (Color online) Calculated excitation rate constants for 420.1 and $419.8 \mathrm{~nm}$ Ar emission lines from ground state, $K_{1 a}$ and $K_{2 a}$, respectively, and metastable Ar, $K_{1 m}$, and $K_{2 m}$, respectively. Vertical lines $x_{1}$ and $x_{2}$ indicate $E / N$ matching $E / N$ in Figs. 11 and 10 , respectively.

excitation rates from the ground state, but drastically different excitation rates from the metastable state. It means that in the absence of metastable atoms in the plasma, the line intensities should be the same (the intensity ratio of the two emission lines is $\sim$ unity). However, in the presence of metastable atoms, the $420 \mathrm{~nm}$ intensity could be dramatically larger than the $419 \mathrm{~nm}$ intensity. Therefore, measurement of the relative intensities of the 419 and $420 \mathrm{~nm}$ spectral lines is a convenient indicator of the presence of metastable atoms.

The above calculations have been performed in the limit of low electron and metastable density. Significant amounts of metastable atoms and electrons in the plasma can change the atomic energy level population rate constants and thus change the emission intensities. It is possible, that the increased metastable atom density will decrease the atomic energy level population rate constants as a result of the decrease of the energetic electron density in the EEDF tail due to inelastic processes of electrons with metastable atoms. At the same time, increasing electron density can lead to decreased or increased atomic energy level population rate constants due to relaxation of the EEDF and redistribution of electrons over energies. Decreased atomic energy level population rate constants will take place if the EEDF has less exciting electrons than the EEDF before relaxation and increased rate constants in the opposite case. This means that exact quantitative information on the metastable atom and electron density can only be obtained after analysis of a specific plasma and can depend on $N_{m}$ and $N_{e}$ themselves.

These speculations on the influence of metastable atoms and electrons on emission intensities have been confirmed by modeling. Examples of the modeling results for $420.1 \mathrm{~nm} \mathrm{Ar}$ emission line are shown in Figs. 3-5.

Figure 3 shows decreasing atomic energy level population rate constants from stepwise excitation and simultaneous increasing rate constants from direct excitation of $3 p_{9}$ level due to increasing electron density. This is the result of increasing electron density with energies above the first excitation level due to electron-electron collisions and decreasing electron density with energies between 3 and $6 \mathrm{eV}$ due to relaxation in the EEDF. Increasing metastable atom density 


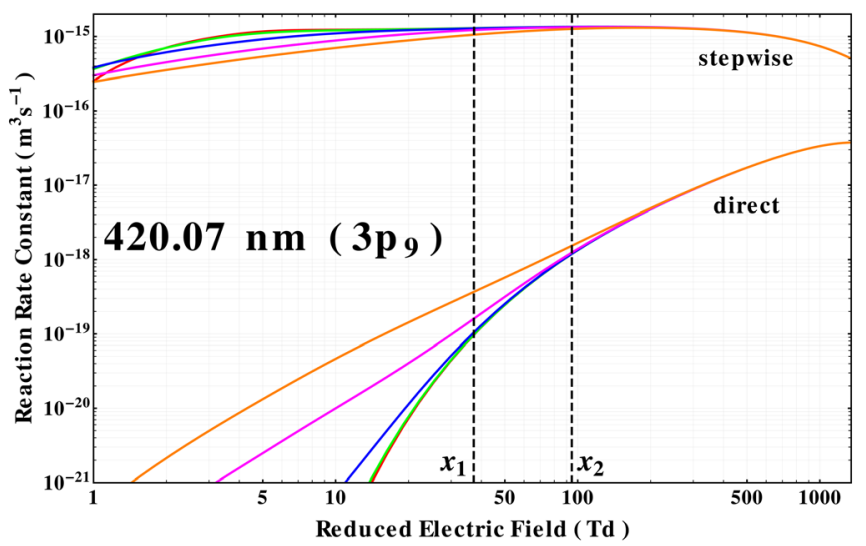

FIG. 3. (Color online) Calculated excitation rate constants for $420 \mathrm{~nm} \mathrm{Ar}$ line: stepwise, $K_{1 m}$ (upper set of 5 curves) and direct, $K_{1 a}$ (lower set of 5 curves) as function of $E / N$. Electron to Ar atom density ratio used are: (lower curves, in ascending order: $N_{e} / N=10^{-8}$ (red), $10^{-6}$ (green), $10^{-5}$ (blue), $10^{-4}$ (magenta), and $10^{-3}$ (orange). Same values of $N_{e} / N$ used for upper curves but in descending order. $N_{m}$ is negligibly small. Vertical lines $x_{1}$ and $x_{2}$ indicate $E / N$ matching $E / N$ in Figs. 11 and 10, respectively.

leads to decreasing emission due to inelastic processes with metastable atoms as shown in Fig. 4. Increasing the electron density reduces this effect as seen in Fig. 5. It is also evident in Figs. 3-5 that as a general rule, the influence of metastable atoms and electrons vanishes for higher $E / N$ which can make discharges with high $E / N$ diagnostically simpler. Although not shown, $K_{2 a}$ and $K_{1 m}$ exhibit similar dependencies on metastable atom and electron densities.

The model confirms the predicted changes of the rate constants due to variation of metastable atom and electron densities. Therefore, any application of emission spectroscopy for plasma diagnostics should take these dependencies into account. However, as seen in Figs. 6 and 7, the ratio of the excitation rate constants, $K_{1 a} / K_{2 a}$ and $K_{1 m} / K_{2 m}$, is much less sensitive to presence of metastable atoms and electron than the excitation rate constants themselves (Figs. 3-5).

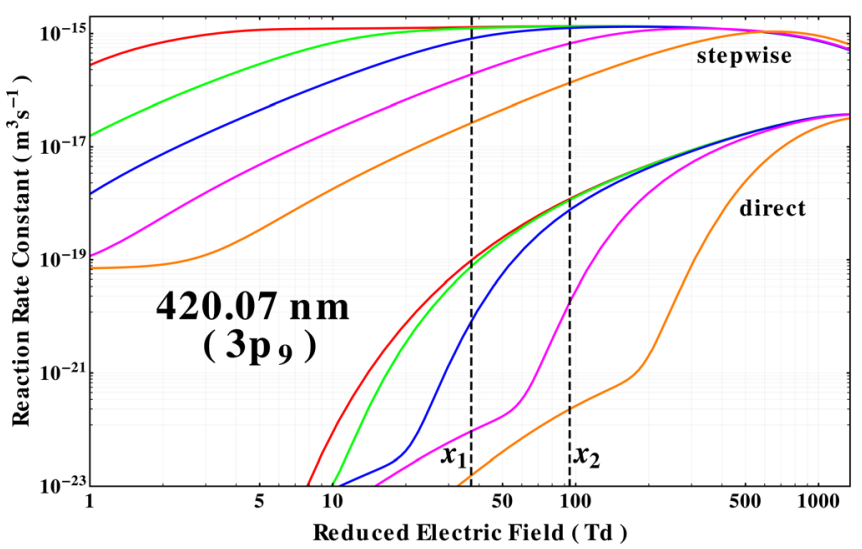

FIG. 4. (Color online) Calculated excitation rate constants for the $420 \mathrm{~nm}$ Ar line: stepwise, $K_{1 m}$ (upper set of 5 curves) and direct, $K_{1 a}$, (lower set of 5 curves) as function of $E / N$. Ar metastable to atom density ratio used for both sets are in descending order: $N_{m} / N=10^{-7}$ (red), $10^{-5}$ (green), $10^{-4}$ (blue), $10^{-3}$ (magenta), and $10^{-2}$ (orange). $N_{e}$ is negligibly small. Vertical lines $x_{1}$ and $x_{2}$ indicate $E / N$ matching to those in Figs. 11 and 10 correspondingly.

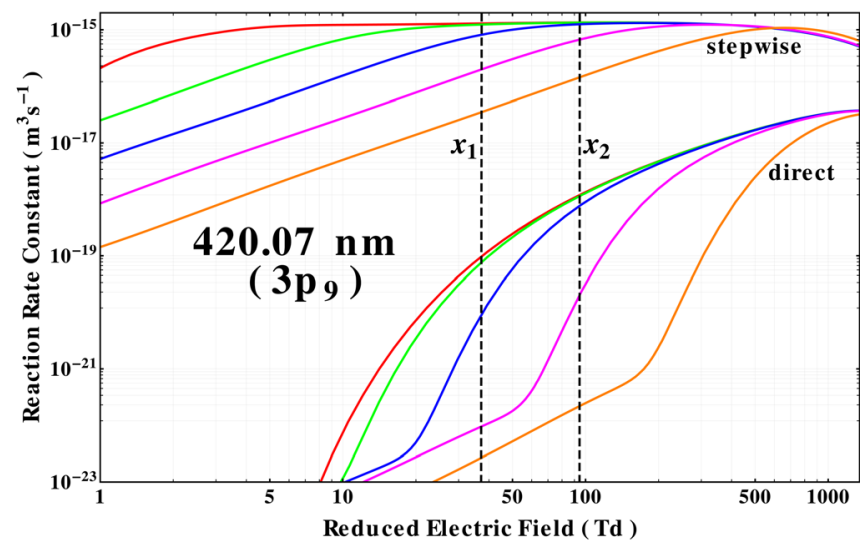

FIG. 5. (Color online) Calculated excitation rate constants for $420 \mathrm{~nm} \mathrm{Ar}$ line: stepwise, $K_{1 m}$ (upper set of 5 curves) and direct, $K_{1 a}$, (lower set of 5 curves) as function of $E / N$. Ar metastable to atom density ratio used for both sets are in descending order: $N_{m} / N=10^{-7}$ (red), $10^{-5}$ (green), $10^{-4}$ (blue), $10^{-3}$ (magenta), and $10^{-2}$ (orange). $N_{m}=10 N_{e}$. Vertical lines $x_{1}$ and $x_{2}$ indicate $E / N$ matching $E / N$ in Figs. 11 and 10, respectively.

It is seen in the figures that, to within a few percent, the excitation rate constants from the ground atomic state ratio, $K_{1 a} / K_{2 a}$, is always close to unity for $E / N<700 \mathrm{Td}$ while for higher $E / N$, the ratio is less than unity. However, the excitation rate constants from the metastable state ratio, $K_{1 m} / K_{2 m}$ varies between 4 and 8 . In summary, it can be generally stated that the metastable atoms and electrons influence the rate constant ratios more at lower reduced electric fields than at higher $E / N$ and the influence vanishes at the higher electric fields. This may be important, for example, in rf plasmas similar to studied in Ref. 16.

\section{GLOBAL MODEL OF INITIAL STAGE OF PULSED DC DISCHARGE}

The temporal variation of the metastable atom and electron plasma densities is described by a non-linear system of kinetic balance equations, ${ }^{1,21}$ which, in general, can only be

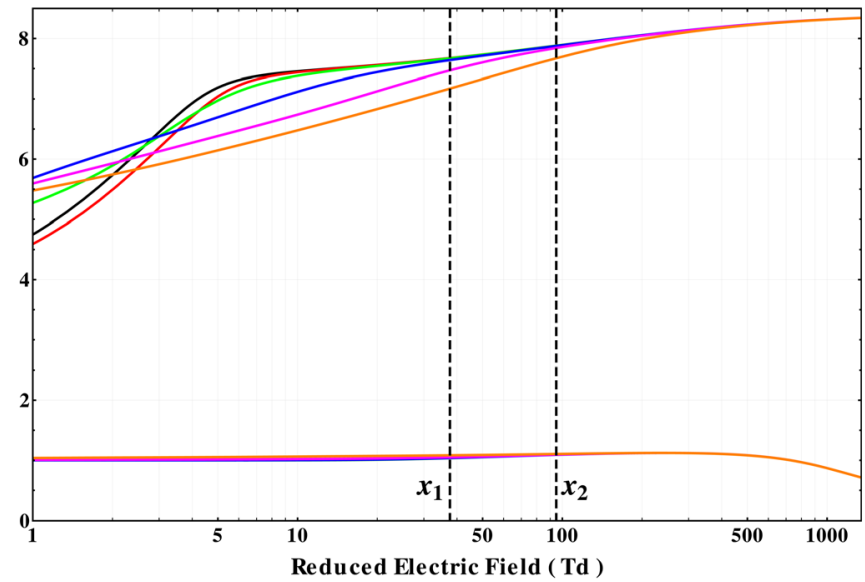

FIG. 6. (Color online) Calculated ratios of excitation rate constants $K_{1 m} / K_{2 m}$ (upper set of 6 curves) and $K_{1 a} / K_{2 a}$ (lower set of 6 curves) as function of $E / N$. Electron to Ar atom densities used are in descending order: $N_{e} / N=0$ (black), $10^{-8}$ (red), $10^{-6}$ (green), $10^{-5}$ (blue), $10^{-4}$ (magenta), and $10^{-3}$ (orange). $N_{m}$ is negligibly small. Vertical lines $x_{1}$ and $x_{2}$ indicate $E / N$ matching $E / N$ in Figs. 11 and 10, respectively. 


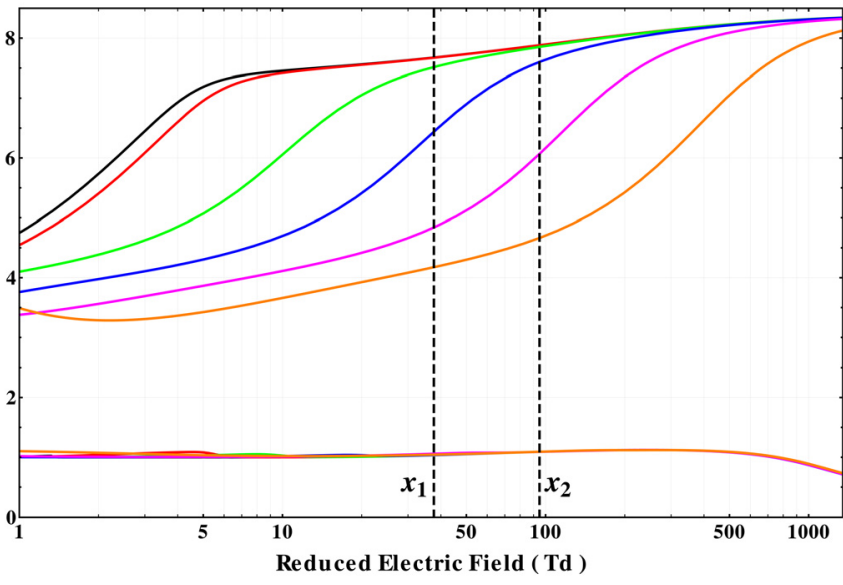

FIG. 7. (Color online) Calculated ratios of excitation rate constants $K_{2 m} / K_{1 m}$ (upper set of 6 curves) and $K_{1 a} / K_{2 a}$ (lower set of 6 curves) as function of $E / N$. Electron to $\mathrm{Ar}$ atom densities used are in descending order: $N_{e} / N=0$ (black), $10^{-8}$ (red), $10^{-6}$ (green), $10^{-5}$ (blue), $10^{-4}$ (magenta), and $10^{-3}$ (orange). $N_{m}=10 N_{e}$. Vertical lines $x_{1}$ and $x_{2}$ indicate $E / N$ matching $E / N$ in Figs. 11 and 10, respectively.

solved numerically. In this section, a global model of nonlinear system of balance equations is presented and applied to the initial stage of a pulsed, rare gas dc discharge. Analysis of the plasma under the specific conditions present during the initial development of the active discharge allows us to simplify the system of equations and obtain an analytic solution. ${ }^{11,12,22}$ The analytical solution may be more convenient than comprehensive numerical modeling for analysis of the general behavior of plasma particles.

It is possible to show that at the initial stage of the discharge, the time-dependant kinetic balance equations may be reduced to the following simple system:

$$
\frac{d N_{e}}{d t}=K_{i} N_{e} N+K_{s w} N_{e} N_{m}-\frac{N_{e}}{\tau_{D}},
$$

and

$$
\frac{d N_{m}}{d t}=K_{e x c} N N_{e}-K_{q} N_{e} N_{m}
$$

Here, $K_{i}$ and $K_{s w}$ are rate coefficients for ionization from the ground and metastable states, respectively, $K_{\text {exc }}$ is the rate coefficient for direct excitation to the metastable level(s), $K_{q}$ is the rate coefficient for metastable quenching by electrons, and $\tau_{D}$ is the diffusion time for electrons. Details of the conditions which lead to the above simplification can be found in Refs. 11 and 12.

In the case of using the emission from Ar to determine metastable atom and electron densities, Eqs. (1) and (2) should be complimented with the following equations for atomic emitting states:

$$
\frac{d N_{1}}{d t}=K_{1 a} N_{e} N+K_{1 m} N_{e} N_{m}-A_{1} N_{1}
$$

and

$$
\frac{d N_{2}}{d t}=K_{2 a} N_{e} N+K_{2 m} N_{e} N_{m}-A_{2} N_{2}
$$

where $N_{1}$ and $N_{2}$ are the densities of atoms in states $3 \mathrm{p}_{9}$ and $3 \mathrm{p}_{5}$, respectively. Taking into account that excited atom densities are in quasi-equilibrium states, it can be shown that line intensities are proportional to $K_{1 a} N_{e} N+K_{1 m} N_{e} N_{m}$ and $K_{2 a} N_{e} N+K_{2 m} N_{e} N_{m}$, respectively, for 420.1 and $419.8 \mathrm{~nm}$ Ar emission lines.

The rate coefficients in Eqs. (1) and (2) depend on the form of the EEDF. Therefore, in general, the EEDF needs to be known as well as the corresponding cross-sections for those processes. However, using simple approximations for these coefficients ${ }^{11}$ and solving Eqs. (1) and (2), it is possible to investigate the general behavior of $N_{e}$ and $N_{m}$ during the initial stage of the discharge. From the general behavior of $N_{e}$ and $N_{m}$, the time between application of electric field and the fast growth of $N_{e}$ and $N_{m}$ can be determined and explain the observed experimental temporal dependencies.

If it is assumed that there is no electronic metastable quenching $\left(K_{q}=0\right)$, Eqs. (1) and (2) can be solved analytically as follows. Let $a=K_{i} N-1 / \tau_{D}, b=K_{s w}, c=K_{\text {exc }} N$ and $\Delta=-\left(a+b N_{m 0}\right)^{2}+2 b c N_{e 0}$. Here, $N_{m 0}$ and $N_{e 0}$ are the metastable and electron densities at the initial part of the active discharge phase (following breakdown). Then, for $\Delta \geq 0$

$$
\begin{gathered}
N_{m}(t)=-\frac{a}{b}+\frac{\sqrt{\Delta}}{b} \cot \left[\frac{\sqrt{\Delta}}{2}(\tau-t)\right], \\
N_{e}(t)=\frac{\Delta}{2 b c} \csc ^{2}\left[\frac{\sqrt{\Delta}}{2}(\tau-t)\right],
\end{gathered}
$$

where

$$
\tau=\frac{2}{\sqrt{\Delta}} \sin ^{-1} \sqrt{\frac{\Delta}{2 c b N_{e 0}}} .
$$

For $\Delta<0$, one simply replaces $\Delta$ with $|\Delta|$ and replaces the circular functions with their hyperbolic equivalents.

Fig. 8 illustrates the relative behavior of $N_{e}$ and $N_{m}$ calculated from Eqs. (5) and (6). Basically the growth of both, $N_{e}$ and $N_{m}$, has two stages: a slow increasing of density after application of the electric field (slow stage) followed by a rapid growth (fast stage). However, depending on the reduced electric field, the metastable slow stage may be longer for higher electric fields or shorter, for lower electric fields as seen in Fig. 8 than for electrons.

This is because variation $K_{i}$ and $K_{\text {exc }}$ with respect to the reduced electric field. The variation of $K_{i}$ and $K_{e x c}$ with $E / N$ is shown in Fig. 9.

For lower reduced electric fields, $E / N, K_{i}$ is smaller than $K_{\text {exc }}$ and for higher electric fields, the situation is reversed. Note, that in the fast stage, the main ionization mechanism may be stepwise ionization for lower electric fields or direct excitation for higher electric fields, whereas in the slow stage it is direct ionization. Thus, the global model demonstrates that the dynamics of $N_{e}$ and $N_{m}$ behavior can be altered by regulation of $E / N$. Temporally resolved emission experiments described in the section illustrate this $E / N$ dependent behavior. 


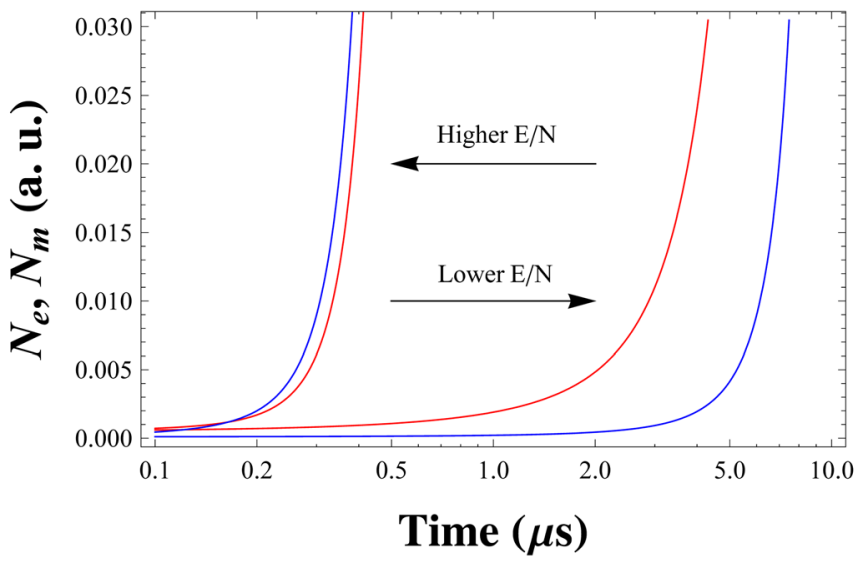

FIG. 8. (Color online) Calculated time variation of metastable atom (red trace) and electron (blue trace) densities (in arbitrary units) in the initial stage of discharge for different $E / N$. For higher E/N, metastable atom lags behind electron density curve while at lower $\mathrm{E} / \mathrm{N}$ the reverse occurs.

\section{EXPERIMENT}

Experiments were performed in a $5 \mathrm{~cm}$ internal diameter glass discharge tube with a cold cathode and the cathode-anode inter-electrode distance was $22 \mathrm{~cm}$. Spectrally pure Ar was used with typical working gas pressures of between 1 and 20 Torr. Measurements were also made with pure $\mathrm{Kr}$ and $\mathrm{Xe}$. The discharge voltage was measured by a high voltage probe, while the discharge current was measured with a Pearson probe current sensor. Plasma emission was imaged perpendicular to the tube axis, mid-way between the cathode and anode, by an optical fiber. The fiber was coupled to the entrance slits of an Acton $0.5 \mathrm{~m}$ spectrometer fitted with an Andor intensified chargecoupled device (ICCD) camera. In Ar time-resolved emission intensity measurements of 419.8 and $420.1 \mathrm{~nm}$ lines were obtained by temporal delay of the camera intensifier gate with respect to the discharge pulse. In Ne spectral lines 345.4 and $347.3 \mathrm{~nm}$ and in Xe spectral lines 467.2 and $480.7 \mathrm{~nm}$ have been used.

\section{RESULTS}

Typical time-resolved spectral and electrical measurements of Ar are shown in Figs. 10 and 11 for high $(3 \mathrm{kV})$ and low $(1 \mathrm{kV})$ applied voltage, respectively.

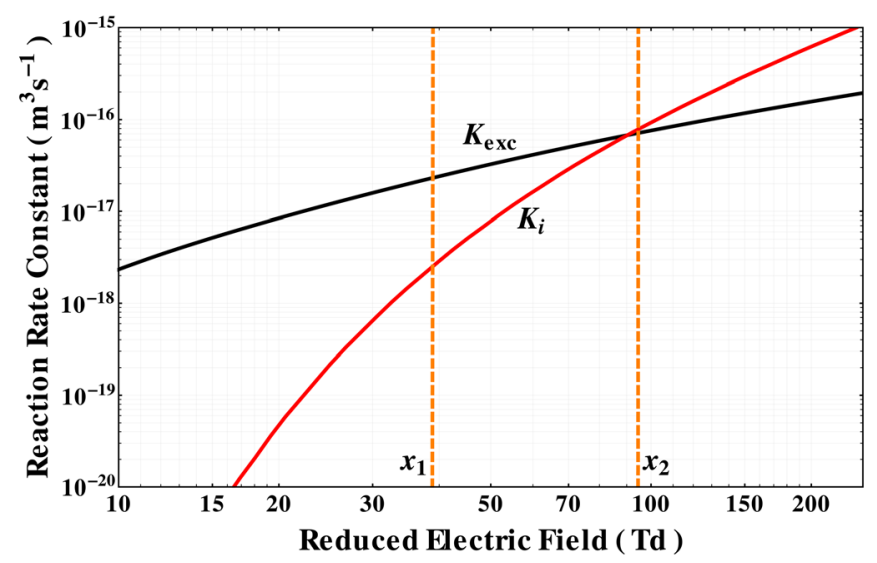

FIG. 9. (Color online) Calculated rate constants $K_{i}$ and $K_{\text {exc }}$ as a function of $E / N$ for Ar. Vertical lines $x_{1}$ and $x_{2}$ indicate $E / N$ matching $E / N$ in Figs. 11 and 10 , respectively.

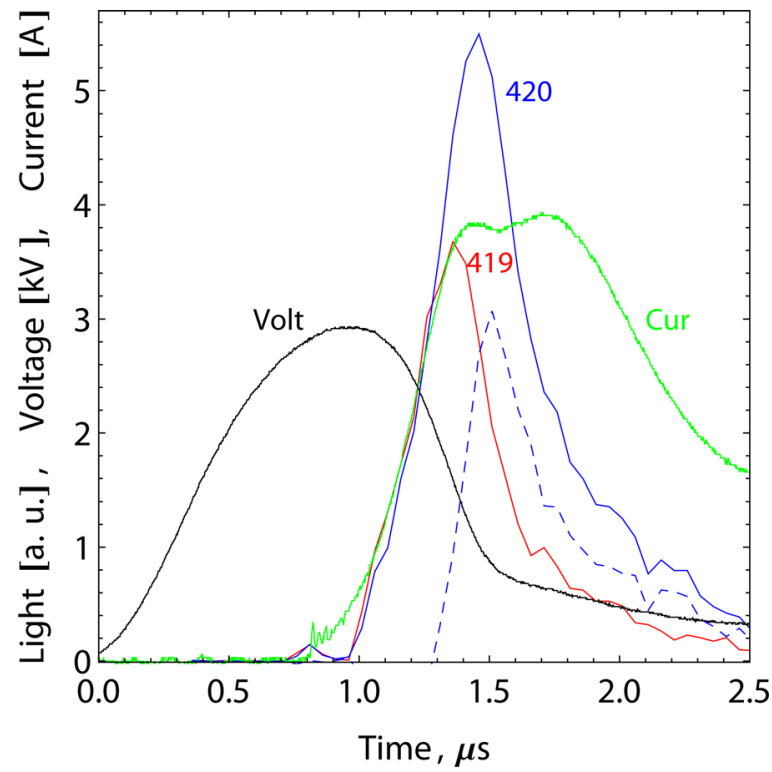

FIG. 10. (Color online) Voltage (black), current (green) and time-resolved emission at $419.8 \mathrm{~nm}$ (red) and $420.1 \mathrm{~nm}$ (blue) Ar lines in 5 Torr, $3 \mathrm{kV}$ applied voltage pulsed dc discharge. $419.8 \mathrm{~nm} \mathrm{Ar}\left(5 \mathrm{p}[1 / 2]_{0} \rightarrow 4 \mathrm{~s}[3 / 2]_{1}\right)$ terminates on non-metastable state and $420.1 \mathrm{Ar}\left(5 \mathrm{p}[5 / 2]_{3} \rightarrow 4 \mathrm{~s}[3 / 2]_{2}\right)$ terminates on lower metastable state. Dashed trace is the difference between temporal response of 420 and $419 \mathrm{~nm}$ spectral lines.

From the voltage (black trace) and current (green trace) traces of Figs. 10 and 11, it is evident that discharge current growth and gas break-down is delayed with respect to the application of the voltage pulse. The delay time depends on the reduced electric field $E / N$ in the gas. The current rise and gas break-down occurs at a longer time-delay for lower voltage (Fig. 11) than for higher voltage (Fig. 10). Reducing the electric field or increasing the gas pressure increases the delay time.

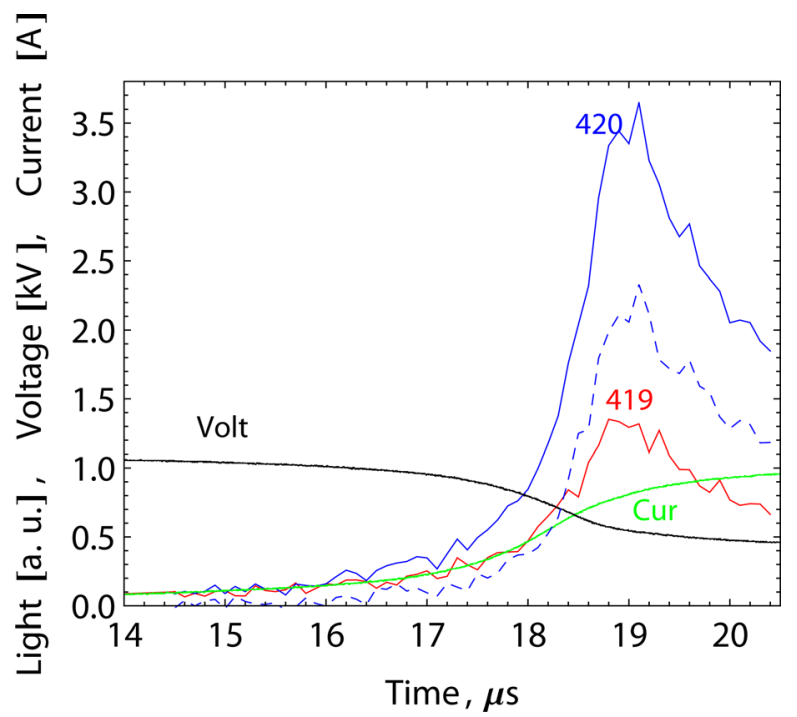

FIG. 11. (Color online) Voltage (black), current (green), and time-resolved emission at $419.8 \mathrm{~nm}$ (red) and $420.1 \mathrm{~nm}$ (blue) Ar lines in 5 Torr, $1 \mathrm{kV}$ applied voltage pulsed de discharge. $419.8 \mathrm{~nm} \operatorname{Ar}\left(5 \mathrm{p}[1 / 2]_{0} \rightarrow 4 \mathrm{~s}[3 / 2]_{1}\right)$ terminates on non-metastable state and $420.1 \mathrm{Ar}\left(5 \mathrm{p}[5 / 2]_{3} \rightarrow 4 \mathrm{~s}[3 / 2]_{2}\right)$ terminates on lower metastable state. Dashed trace is the difference between temporal response of 420 and $419 \mathrm{~nm}$ spectral lines. 


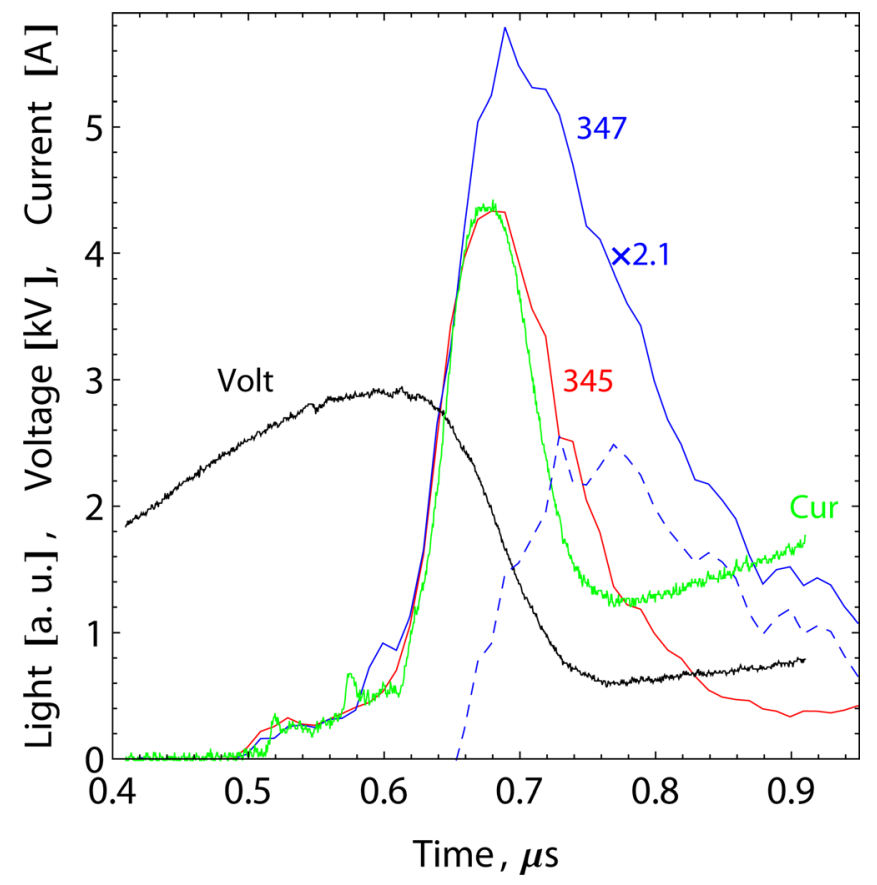

FIG. 12. (Color online) Voltage (black) and current (green) and time resolved emission at $347.3 \mathrm{~nm}$ (blue) and $345.4 \mathrm{~nm}$ (red) Ne lines in $1 \mathrm{Torr}, 3 \mathrm{kV}$ applied voltage in pulsed dc discharge. $345 \mathrm{~nm} \mathrm{Ne}\left(4 \mathrm{p}[1 / 2]_{0} \rightarrow 3 \mathrm{~s}[3 / 2]_{1}\right)$ terminates on non-metastable state and $347 \mathrm{~nm} \mathrm{Ne}\left(4 \mathrm{p}[5 / 2]_{3} \rightarrow 3 \mathrm{~s}[3 / 2]_{2}\right)$ terminates on lower metastable state. Dashed trace is the difference between temporal response of 347 and $345 \mathrm{~nm}$ spectral lines.

The temporal behavior of the emission intensity of $419 \mathrm{~nm}$ line (red trace) coincides closely with the current trace up to the emission maximum for both high and low applied voltages. The $419 \mathrm{~nm}$ emission originates from the Ar $3 p_{5}$ level, produced mainly from direct electron excitation and follows the electron current, $e N_{e} \mu E$, where $e$ is the electron charge, $\mu$ is the electron mobility, and $E$ is the electric field. Thus, the $419 \mathrm{~nm}$ emission is proportional to the electron density growth. On the other hand, the behavior of $420 \mathrm{~nm}$ emission, originating from the $3 \mathrm{p}_{9}$ level, is much more sensitive to the presence of metastable atoms and depends on the reduced electric field. For smaller electric fields (Fig. 11), the $420 \mathrm{~nm}$ emission rise precedes the current growth while for the higher field (Fig. 10), emission rise coincides with the current (and emission of $419 \mathrm{~nm}$ line) growth. In the last case, it means that metastable atom density fast growth occurs after the electron density fast growth in contrast to the small electric field case. This is more easily seen in the difference of the emission lines intensity (dashed trace). Here, the difference in emission is clearly delayed with respect to the rise in current for higher $E / N$ (Fig. 10) than for lower $E / N$ (Fig. 11). The difference in emission is representative of the behavior of the metastable atom density. Both spectral lines are equally sensitive to the presence of electrons (their ratio is $\sim$ unity when the metastable density is low); however, $420 \mathrm{~nm}$ line is much more sensitive to the presence of metastable atoms $(420 \mathrm{~nm}$ emission is greater than $419 \mathrm{~nm}$ emission in the presence of substantial amounts of metastable atoms). Thus, the $E / N$ dependence of the duration of the slow stages transition for metastable atoms and electrons (shown in Fig. 8) is confirmed experimentally.

It is also possible to obtain quantitative information about metastable atom density from these measurements. Some information about obtain metastable atom density has been previously presented by Adams et al. ${ }^{18,19}$ and will be published in more detail elsewhere. For an approximate evaluation of $N_{m}$, the Ar spectral lines 419.8 and $420.1 \mathrm{~nm}$ intensity ratios and Figs. 2-7 can be used. This ratio is equal to $\left(K_{2 a}+K_{2 m} N_{m} / N\right) /\left(K_{1 a}+K_{1 m} N_{m} / N\right)$ and for the condition investigated $K_{1 a} \approx K_{2 a}$. As an example, using values of $K_{1 m}, K_{2 m}$, and $K_{1 a}$ from Fig. 2 , the metastable atom density is determined to be roughly $2 \times 10^{12} \mathrm{~cm}^{-3}$ at $\sim 19 \mu$ s from the line intensities in Fig. 11.

Similar pairs of emission lines can be identified in other rare gases. In $\mathrm{Ne}$, the transition lines are 345.4 and $347.3 \mathrm{~nm}$ and in Xe they are 480.7 and $467.2 \mathrm{~nm}$, respectively. Thus, by measuring the temporal behavior of the corresponding emission lines, changes in the metastable atom and electron density can be determined for these other rare gases. Voltage, current, and emission measurements in $\mathrm{Ne}$ and Xe were conducted. Typical measurements in neon for high and low applied voltages $(E / N)$ are shown in Figs. 12 and 13 .

It is seen from those figures that the time-resolved emission lines exhibit very similar behavior with respect to $E / N$, compared with the emission in Ar. The metastable atom and electron density growth behaves differently, depending on the reduced electric field. This also confirms the theoretical prediction shown in Fig. 8. However, in order to evaluate the $\mathrm{Ne}$ metastable atom density, emission from $347 \mathrm{~nm}$ Ne line must be increased by a factor of 2.1 so that the emission

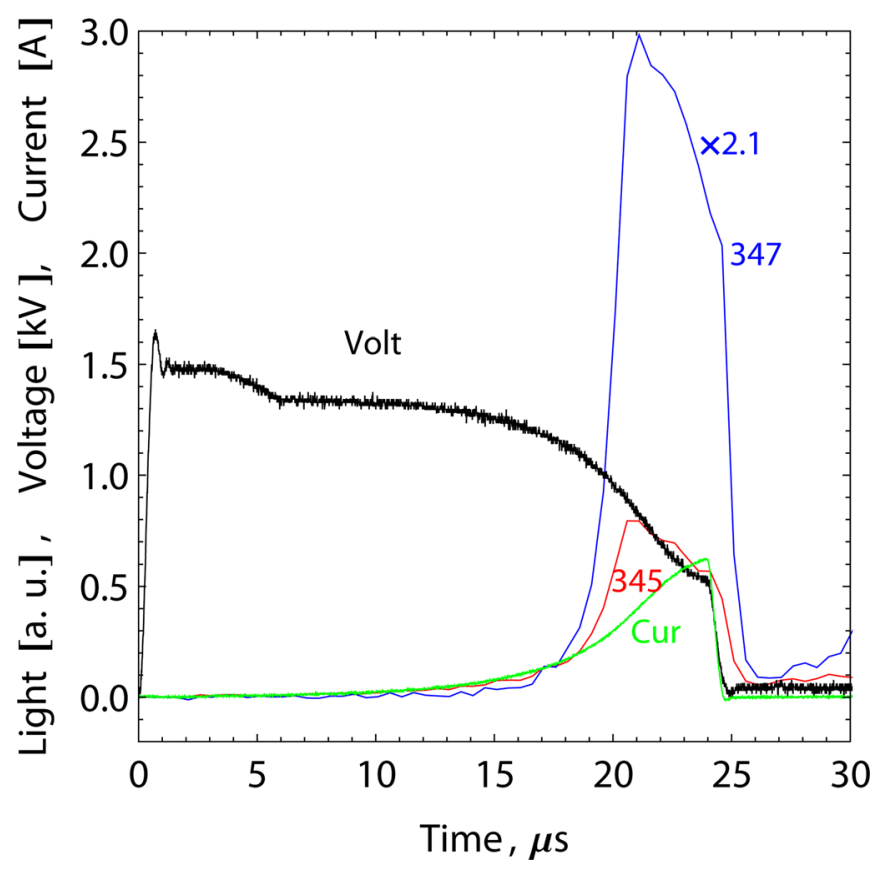

FIG. 13. (Color online) Voltage (black) and current (green) and time resolved emission at $347.3 \mathrm{~nm}$ (blue) and $345.4 \mathrm{~nm}$ (red) Ne lines in 20 Torr, $1.5 \mathrm{kV}$ applied voltage in pulsed dc discharge. $345 \mathrm{~nm} \mathrm{Ne}\left(4 \mathrm{p}[1 / 2]_{0} \rightarrow 3 \mathrm{~s}[3 / 2]_{1}\right)$ terminates on non-metastable state and $347 \mathrm{~nm} \mathrm{Ne}\left(4 \mathrm{p}[5 / 2]_{3} \rightarrow 3 \mathrm{~s}[3 / 2]_{2}\right)$ terminates on lower metastable state. Dashed trace is the difference between temporal response of 347 and $345 \mathrm{~nm}$ spectral lines. 


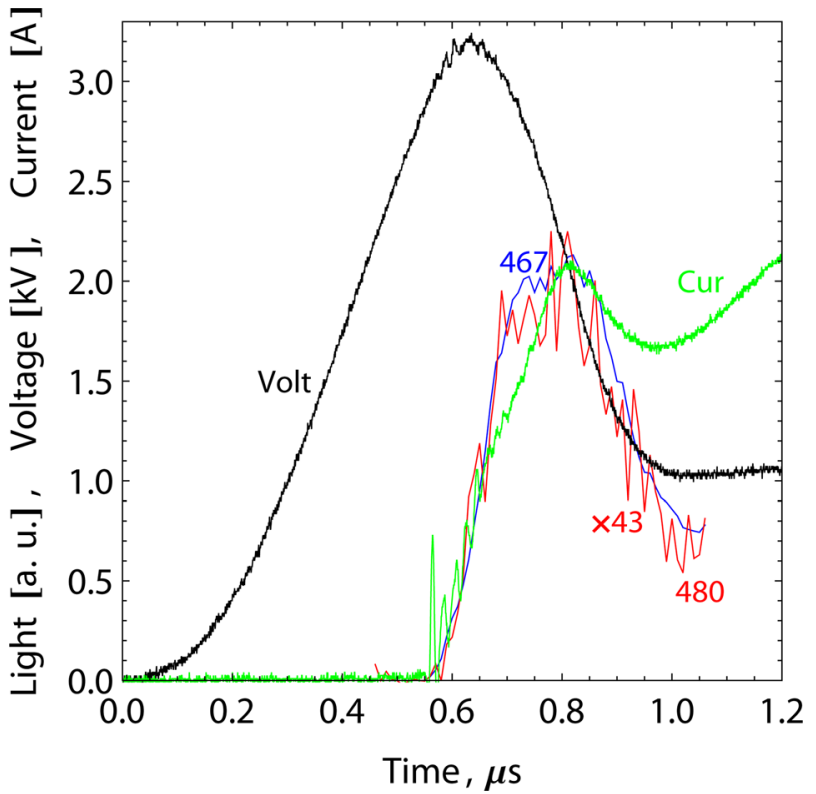

FIG. 14. (Color online) Voltage (black) and current (green) and time resolved emission at $480.7 \mathrm{~nm}$ (red) and $467.2 \mathrm{~nm}$ (blue) $\mathrm{Xe}$ lines in 2Torr, 5kV applied voltage in pulsed dc discharge. $480.7 \mathrm{~nm} \mathrm{Xe}\left(7 \mathrm{p}[1 / 2]_{0} \rightarrow 6 \mathrm{~s}[3 / 2]_{1}\right)$ terminates on non-metastable state and $467.2 \mathrm{~nm} \mathrm{Xe}\left(7 \mathrm{p}[5 / 2]_{3} \rightarrow 6 \mathrm{~s}[3 / 2]_{2}\right)$ terminates on lower metastable state. Dashed trace is the difference between temporal response of 467.2 and $480.7 \mathrm{~nm}$ spectral lines.

intensity is comparable to $345 \mathrm{~nm}$ emission of $345 \mathrm{~nm}$ for the low metastable density case. This would suggest that the cross-section for direct excitation of corresponding excited $\mathrm{Ne}$ levels is correspondingly different. With 2.1 scaling factor, the metastable atoms behavior can be evaluated, similar to the $\mathrm{Ar}$ case, with subtraction of the two emission line intensities (shown in Fig. 12 by a dashed curve).

Measurements in Xe show similar corresponding spectral lines and metastable atom and electron densities behavior. Note, however, that in this case the emission line multiplicative factor for metastable atom behavior comparison is 43 . An example of emission measurements in Xe for higher electric field is shown in Fig. 14.

\section{CONCLUSIONS}

Measurements of emission intensities of spectral lines pairs, 419.8 and $420.1 \mathrm{~nm}$ in Ar or corresponding lines in other noble gases, may be a simple, convenient method to measure metastable atom and electron densities dynamics in plasmas. These emission intensity measurements experimentally demonstrated that in the initial stage of a pulsed dc dis- charge, the metastable state atoms and electrons behave differently, depending on the reduced electric field applied to the discharge. This difference in behavior can be used in technological applications for regulating plasma properties.

\section{ACKNOWLEDGMENTS}

The authors would like to thank C. A. DeJoseph, Jr., I. Kaganovich, and Y. Raitses for valuable discussions and J. Miles for help in experiments. This work was supported by the DOE OFES (Contract No. DE-SC0001939), NSF (Grant No. CBET-0903635), GK 14.740.11.0893 and AFOSR.

${ }^{1}$ M. A. Lieberman and A. J. Lichtenberg, Principles of Plasma Discharges and Materials Processing (Wiley, New York, 1994).

${ }^{2}$ F. F. Chen and J. P. Chang, Lecture Notes on Principles of Plasma Processing (Kluwer, New York, 2003).

${ }^{3}$ P. Yeates and E. T. Kennedy, Phys. Plasmas 18, 063106 (2011).

${ }^{4}$ M. A. Song, Y. W. Lee, and T. H. Chung, Phys. Plasmas 18, 023504 (2011).

${ }^{5}$ N. Konjevic, S. Jovicevic, and M. Ivkovic, Phys. Plasmas 16, 103501 (2009).

${ }^{6}$ E. M. Hollman and A. Yu. Pigarov, Phys. Plasmas 9, 4330 (2002).

${ }^{7}$ S. Yun, V. Kolobov, and G. R. Tynan, Phys. Plasmas 8, 3069 (2001).

${ }^{8}$ V. I. Demidov, S. V. Ratynskaia, and K. Rypdal, Rev. Sci. Instrum. 73 3409 (2002).

${ }^{9}$ V. Godyak and V. I. Demidov, J. Phys. D: Appl. Phys. 44, 233001 (2011).

${ }^{10}$ A. Shashurin, J. Li, T. Zhuang, M. Keidar, and I. I. Beilis, Phys. Plasmas 18, 073505 (2011).

${ }^{11}$ A. Kudryavtsev, T. Musienko, and V. Skrebov, J. Phys. D: Appl. Phys. 20, 718 (1987).

${ }^{12}$ V. I. Demidov, C. A. DeJoseph, Jr., and A. A. Kudryavtsev, Plasma Sources Sci. Technol. 13, 600 (2004).

${ }^{13}$ V. I. Demidov, C. A. DeJoseph, Jr., and A. A. Kudryavtsev, IEEE Trans. Plasma Sci. 34, 825 (2006).

${ }^{14}$ Workshop: Control of Distribution Functions in Low Temperature Plasma, edited by I. Kaganovich, Y. Raitses, D. Graves, and G. Oehrlein (American Physical Society, College Park, MD [Bull. Am. Phys. Soc. 56(15), 11 (2011)].

${ }^{15}$ H. Shin, W. Zhu, L. Xu, V. M. Donnelly, and D. J. Economou, Plasma Sources Sci. Technol. 20, 055001 (2011)

${ }^{16}$ C. A. DeJoseph, Jr., and V. I. Demidov, J. Phys. B 38, 3805 (2005).

${ }^{17}$ R. O. Jung, J. B. Boffard, L. W. Anderson, and C. C. Lin, Phys. Rev. A 75, 052707 (2007).

${ }^{18}$ S. Adams, J. Miles, A. Laber, V. Demidov, J. Williamson, and B. Tolson, in 19th International Symposium on Plasma Chemistry, Bochum (July 26-31, 2009)

${ }^{19}$ S. Adams, J. Miles, L. Sobota, J. Caplinger, V. Demidov, and J. Williamson, in 20th International Symposium on Plasma Chemistry, Philadelphia (July 24-29, 2011)

${ }^{20}$ E. A. Bogdanov, C. A. DeJoseph, Jr., V. I. Demidov, A. A. Kudrayvtsev, and K. Yu. Serditov, Plasma Sources Sci. Technol. 16, 697 (2007).

${ }^{21}$ T. Fujimoto, J. Phys. Soc. Jpn. 47, 265 (1979)

${ }^{22}$ V. Demidov, T. Rudakova, S. Rytenkov, and V. Skrebov, J. Phys. B 24, 4281 (1991). 